\title{
On a Generalization of the Index Notation for Absolute Tensors of Arbitrary Order
}

\author{
Edmund H. Brown
}

(January 7, 1960)

\begin{abstract}
A generalized vector index notation is introduced which facilitates study of properties of tensors regardless of order and variance of components. This generality is obtained by replacing the set of $m$ indices, each of which vary in a single domain of $n$ integers, by one vector index which varies over the integral lattice points of an $m$-dimensional domain. The notation also suggests an elegant treatment of order transformations of tensors.
\end{abstract}

\section{Introduction}

The usual index notation for the components of a tensor in a given coordinate system has the advantage that repetitious display of similar components of tensor quantities or equations is avoided without sacrificing directness and simplicity of algebraic manipulations. At the same time, retention of a single base symbol (to which the indices are attached) for each tensor is conceptually appealing since there is a one-to-one correspondence between base symbols and covariant objects. This correspondence is especially desirable in the expression of physical theories since physical observables are required to have the covariance property. A disadvantage of the notation is that, in the study of general properties of tensors, equations such as the transformation relations, the expressions for covariant derivatives, or the integrability conditions do not retain the same form, but require an increasing number of terms or factors with increasing tensor order. In this note, an amusing generalization of the usual notation is presented which permits a study of such general tensor properties in a form which is invariant with respect to order. In addition, this generalization suggests some new concepts, such as that of the order transformation.

\section{Index Notation}

Let $T$ be an $m$ th order tensor defined on an $n$-dimensional coordinate-space $G$ and $x^{2}$ be an arbitrary coordinate system in $G$. Then, $m$ indices $j_{a}$ may be defined in the domain $J\left(_{1}\right)$ consisting of the set of positive integers such that $0<j_{a} \leq n$ for $a=1,2, \ldots m$. By assigning one component of $T$ in the coordinate system $x^{i}$ to each set of the indices $j_{a}$ the tensor $T$ may be completely specified and written in the index notation as $t^{j_{1} j_{2}} \ldots j_{m}$.

For every coordinate transformation in $G$ given by a set of $n$ analytic functions $x^{s}=x^{s}\left(x^{i}\right)$ there will be a transformation of the components of the tensor $T$ at a point $x^{i}$ given by

$$
t^{s_{1} s_{2}} \ldots s_{m}=t^{j_{1} j_{2}} \ldots j_{m} x_{j_{1}}^{s_{1}} x_{j_{2}}^{s_{2}} \ldots x_{j_{m}}^{s_{m}},
$$

where

$$
x_{j_{a}}^{s_{a}}=\frac{\partial x^{s_{a}}}{\partial x^{j_{a}}}
$$

Thus, for a vector, $m=1$, the transformation law will be

$$
t^{s}\left(x^{r}\right)=t^{j}\left(x^{i}\right) x_{j}^{s}
$$

and, in general, the transformation law of an $m$ th order tensor will involve exactly $m$ of the transformation matrix factors $x_{r}^{i}$. 
If a tensor field $T(G)$ is defined on all points of $G$, the true rate of change of $T$ is given by the covariant derivative

$$
\left.t^{j_{1} j_{2} \ldots j_{m}}\right|_{k}=\frac{\partial t^{j_{1} j_{2} \ldots j_{m}}}{\partial x^{k}}+t^{h j_{2} \ldots j_{m}} \Gamma_{\cdot h k}^{j_{1}}+t^{j_{1} h \ldots j_{m}} \Gamma_{\cdot h k}^{j_{2}}+\cdots+t^{j_{1} j_{2} \ldots h} \Gamma_{\cdot h k}^{j_{m}},
$$

where the convenient vertical bar notation of Green and Zerna ${ }^{1}$ is used. For a vector, the expression for the covariant derivative becomes merely

$$
\left.t^{i}\right|_{k}=\frac{\partial t^{1}}{\partial x^{k}}+t^{h} \Gamma_{\cdot h k}^{i}
$$

and, in general, the covariant derivative of an $m$ th order tensor will involve exactly $m$ "corrective" terms in the Christoffel Connection $\Gamma_{. j k}^{i}$.

As a final example, the integrability conditions for an $m$ th order tensor may be written

$$
\left.t^{i_{1} i_{2}} \ldots i_{m}\right|_{k j}-t^{\left.i_{1} i_{2} \ldots i_{m}\right|_{\jmath k}}=t^{h i_{2} \ldots i_{m}} R_{\cdot h \mid j k}^{i_{1}}+t^{i_{1} h \ldots i_{m}} R_{\cdot h \mid j k}^{i_{2}}+\ldots+t^{i_{1} i_{2}} \ldots h R_{\cdot h \mid j k}^{i_{m}}
$$

where $R_{\cdot j \mid k h}^{i}$ is the Riemann-Christoffel curvature tensor. For a vector, the integrability condition reduces to

$$
\left.t^{i}\right|_{k j}-\left.t^{i}\right|_{j k}=t^{h} R_{\cdot h \mid j k}^{i}
$$

and, in general, there will be exactly $m$ terms on the right side of the integrability conditions (3.m) for every $m$ th order tensor.

\section{Index Vector}

In the previous section, exactly $m$ indices $j_{a}$ were adjoined to the base symbol of an $m$ th order tensor, with each of the $j_{a}$ varying in a single domain $J_{(1)}$. We now consider the converse possibility of a Cartesian lattice index space $J_{(m)}$ which is the $m$ th Cartesian product of $m$ of the spaces $J_{(1)}$ and define a Cartesian index vector $\mathbf{j}$ in this space. For the moment, we consider only index vectors whose components are positive integers, that is, we restrict $J_{(m)}$ to the first hyperquadrant. For an $m$ th order tensor on an $n$-dimensional space, a maximum index vector $\hat{\mathbf{j}}$ can be defined by the relations $\hat{j}_{a}=n, a=1, \ldots, m$, for its components $\hat{j}_{a}$. Then, since there will be exactly $n^{m}$ index vectors $\mathbf{j}$ which satisfy the conditions $0<j_{a} \leq \hat{j}_{a}$, one component of the tensor may be assigned to each of index vectors $\mathbf{j}$ and the tensor itself represented by the symbol $t^{\mathrm{j}}$.

One of the simplest methods of forming higher-order tensors is by adjunction of vectors. Since coordinate differentials are vectors, a first application of this index vector notation results in the symbol $d x^{\mathbf{j}}=d x^{j_{1}} d x^{j_{2}} \ldots d x^{j_{m}}$. Although the transformation matrices $x_{j}^{s}$ do not represent tensors, the symbol $x_{\mathbf{j}}^{\mathbf{s}}=x_{j_{1}}^{s_{1}} x_{j_{2}}^{s_{2}} \ldots x_{j_{m}}^{s_{m}}$ may be constructed by analogy with $d x^{\mathbf{j}}$. Using this generalized matrix, the transformation law for a tensor of arbitrary order may be written

$$
t^{\mathbf{s}}=t^{\mathbf{j}} x_{\mathbf{j}}^{\mathbf{s}}
$$

where (corresponding to the usual summation convention) the diagonal repetition of $\mathbf{j}$ indicates summation over all the index vectors in $J_{(m)}$.

By analogy with the derivation of Brillouin, ${ }^{2}$ generalized expressions for the Christoffel Connection may be obtained by considering the displacement of a tensor of arbitrary order. If $x^{a}$ are normal coordinates, that is, coordinates such that the covariant differential $D t^{\mathbf{a}}$ is equal to the ordinary differential $d t^{\mathbf{a}}$ in the infinitesimal neighborhood of the point $x^{b}$, then

$$
t^{\mathbf{a}}\left(x^{b}+d x^{b}\right)=t^{\mathbf{a}}\left(x^{b}\right)+D t^{\mathbf{a}}
$$

\footnotetext{
1 A. E. Green and W. Zerna, Theoretical elasticity (Oxford University Press, Oxford, England, 1954).

2 L. Brillouin, Les tenseurs en mécanique et en élasticité (Dover Publications, New York, N.Y., 1946).
} 
Transforming to arbitrary coordinates $x^{i}$ by means of the transformation matrices $x_{\mathbf{a}}^{\mathbf{i}}\left(x^{b}+d x^{b}\right)$ and $x_{\mathbf{a}}^{\mathbf{i}}\left(x^{b}\right)$ gives

$$
\begin{aligned}
t^{\mathbf{i}}\left(x^{k}\right)=t^{\mathbf{a}}\left(x^{b}\right) x_{\mathbf{a}}^{\mathbf{i}}\left(x^{b}\right), \quad t^{\mathbf{i}}\left(x^{k}+d x^{k}\right)=\left\{t^{\mathbf{a}}\left(x^{b}\right)\right. & \left.+D t^{\mathbf{a}}\right\} x_{\mathbf{a}}^{\mathbf{i}}\left(x^{b}+d x^{b}\right) \\
& =\left\{t^{\mathbf{a}}\left(x^{b}\right)+D t^{\mathbf{a}}\right\}\left\{x_{\mathbf{a}}^{\mathbf{i}}\left(x^{b}\right)+x_{\mathbf{a} b}^{\mathbf{i}} d x^{b}\right\}=t^{\mathbf{i}}\left(x^{k}\right)+D t^{\mathbf{i}}+t^{\mathbf{j}} x_{\mathbf{a} b}^{\mathbf{i}} x_{\mathbf{j}}^{\mathbf{a}} x_{k}^{b} d x^{k},
\end{aligned}
$$

where $x_{\mathbf{a} b}^{\mathbf{i}}=\partial x_{\mathbf{a}}^{\mathbf{i}} / \partial x^{b}$ and where we neglect the term containing the second-order differential, $D t^{\mathbf{a}} x_{\mathbf{a} b}^{\mathbf{i}} d x^{b}$. Defining the Christoffel Connection by $\Gamma_{\cdot \mathbf{j} k}^{\mathbf{i}}=-x_{\mathbf{a} b}^{\mathbf{i}} x_{\mathbf{j}}^{\mathbf{a}} x_{k}^{b}$, the covariant differential can be written

$$
D t^{\mathbf{i}}=d t^{\mathbf{i}}+t^{\mathbf{j}} \Gamma_{\cdot \mathbf{j} k}^{\mathbf{i}} d x^{k}
$$

and the generalization of the expression (2.m) for the covariant derivative of a tensor of arbitrary order becomes

$$
\left.t^{\mathbf{i}}\right|_{k}=\frac{\partial t^{\mathbf{i}}}{\partial x^{k}}+t^{\mathbf{j}} \Gamma_{\cdot \mathbf{j} k}^{\mathbf{i}}
$$

If the normal coordinates $x^{a}$ are assumed to be obtained by transformation from a third set of arbitrary coordinates $x^{r}$, we can write

or,

$$
\Gamma_{\cdot \mathbf{j} k}^{\mathbf{i}}=-\left(x_{\mathbf{r}}^{\mathbf{i}} x_{\mathbf{a}}^{\mathbf{r}}\right)_{b} x_{\mathbf{j}}^{\mathbf{a}} x_{k}^{b}=-x_{\mathbf{a} b}^{\mathbf{r}} x_{\mathbf{p}}^{\mathbf{a}} x_{s}^{b} x_{\mathbf{r}}^{\mathbf{i}} x_{\mathbf{j}}^{\mathbf{p}} x_{k}^{s}-x_{\mathbf{r} \mathbf{s}}^{\mathbf{i}} x_{\mathbf{a}}^{\mathbf{r}} x_{\mathbf{j}}^{\mathbf{a}} x_{b}^{s} x_{k}^{b}
$$

$$
\Gamma_{\cdot \mathbf{j} k}^{\mathbf{i}}=\Gamma_{\cdot \mathbf{p} s}^{\mathbf{r}} x_{\mathbf{r}}^{\mathbf{i}} x_{\mathbf{j}}^{\mathbf{p}} x_{k}^{s}-x_{\mathbf{r} s}^{\mathbf{i}} x_{\mathbf{j}}^{\mathbf{r}} x_{k}^{s},
$$

which is the generalization of the well-known Christoffel transformation law. Clearly, the original definition of $x_{\mathbf{j}}^{\mathbf{s}}$ requires that

$$
t^{\mathbf{j}} \Gamma_{\cdot \mathbf{j} k}^{\mathbf{i}}=t^{j i_{2} \ldots i_{m}} \Gamma_{\cdot \cdot k}^{i_{1}}+t^{i_{1} j} \ldots i_{m} \Gamma_{\cdot j k}^{i_{2}}+\cdots+t^{i_{1} i_{2} \ldots{ }^{j}} \Gamma_{\cdot j k}^{i_{m}},
$$

by the law of differentiation of products and the relation $\delta_{j}^{i}=x_{a}^{i} x_{j}^{a}$.

For an arbitrary tensor field $t^{\mathrm{i}}\left(x^{k}\right)$, the expression for the covariant differential permits a calculation of the change in the components at a fixed point after integration around a given curve $C$ of $G$. If $\delta t^{\mathbf{i}}$ is the change at the point, then

$$
\begin{aligned}
& \delta t^{\mathbf{i}}=\oint_{C} d t^{\mathbf{i}}=\oint_{C} D t^{\mathbf{i}}-\oint_{C} t^{\mathbf{h}} \Gamma_{\cdot \mathbf{h} k}^{\mathbf{i}} d x^{k}=\left.\oint_{C} t^{\mathrm{i}}\right|_{k} d x^{k}-\oint_{C} t^{\mathbf{h}} \Gamma_{\cdot \mathbf{h} k}^{\mathbf{i}} d x^{k} \\
& =\frac{1}{2} \iint_{S}\left\{\frac{\left.\partial t^{\mathrm{i}}\right|_{k}}{\partial x^{j}}-\frac{\left.\partial t^{\mathrm{i}}\right|_{j}}{\partial x^{k}}\right\} d s^{j k}-\frac{1}{2} \iint_{S}\left\{\frac{\partial t^{\mathbf{h}} \Gamma_{\cdot \mathbf{h} k}^{\mathrm{i}}}{\partial x^{k}}-\frac{\partial t^{\mathbf{h}} \Gamma_{\cdot \mathbf{h} j}^{\mathrm{i}}}{\partial x^{k}}\right\} d s^{j k}
\end{aligned}
$$

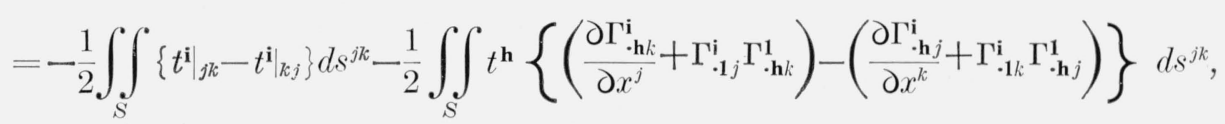

where $S$ is the surface enclosed by $C$ and $d s^{j k}$ the differential surface element. Following the usual definition of the ${ }_{\alpha}^{*}$ curvature tensor,

$$
R_{\cdot \mathbf{h} \mid j k}^{\mathbf{i}}=\left(\frac{\partial \Gamma_{\cdot \mathbf{h} k}^{\mathbf{i}}}{\partial x^{j}}+\Gamma_{\cdot \cdot 1 j}^{\mathbf{i}} \Gamma_{\cdot \mathbf{h} k}^{\mathbf{1}}\right)-\left(\frac{\partial \Gamma_{\cdot \mathbf{h} j}^{\mathbf{i}}}{\partial x^{k}}+\Gamma_{\cdot \cdot 1 k}^{\mathbf{i}} \Gamma_{\cdot \mathbf{h} j}^{1}\right)
$$

so that the change $\delta t^{\mathbf{i}}$ may be written

$$
\delta t^{\mathbf{i}}=-\frac{1}{2} \iint_{S}\left\{\left.t^{\mathbf{i}}\right|_{j k}-\left.t^{\mathbf{i}}\right|_{k j}+t^{\mathbf{h}} R_{\cdot \mathbf{h} \mid j k}^{\mathbf{i}}\right\} d s^{j k} .
$$


Since integrability requires $\delta t^{\mathrm{i}}=0$ for arbitrary area $S$, we arrive at the generalization of the integrability conditions (3.m) in the form

$$
\left.t^{\mathrm{i}}\right|_{k j}-\left.t^{\mathrm{i}}\right|_{j k}=t^{\mathbf{h}} R_{\cdot \mathbf{h} \mid j k}^{\mathbf{i}} \cdot
$$

Thus, to be integrable, a tensor field cannot be completely arbitrary, but must satisfy the conditions (3). In the case of a tensor field obtained by parallel displacement to all points of a tensor given initially at one point, we must have $D t^{i} \equiv 0$; thus, the field can be integrable only if the space is Euclidean, $R_{\cdot h}^{\mathrm{i} \mid j k} \equiv 0$.

\section{Mixed Index Vectors}

The index vector notation facilitates study of general tensor properties regardless of order. In such a study, however, specification of the covariancy or contravariancy of components with respect to particular indices may be even less important, especially in a metric space where components may be immediately transformed from one type of variance to the other. Thus, a general notation should allow interpretation of such differences in variance without actually displaying the differences in its symbols.

Let us consider what significance can be attached to an index space $J_{(m)}$ which includes all hyperquadrants, that is, which allows both positive and negative integers as components of the index vector $\mathbf{j}$. Each hyperquadrant in the enlarged $J_{(m)}$ will be bounded by $m$ hyperplanes formed on $m$ semi-axes and, thus, there will be $2^{m}$ such hyperquadrants in $J_{(m)}$. Defining a maximum index vector $\hat{\mathbf{j}}$ by $\hat{j}_{a}= \pm n$ with some particular signature $\sigma=\{+,+,-,+\ldots\}$ for the signs of the components then, with the restrictions sign $j_{a}=\operatorname{sign} \hat{j}_{a}$ and $0<\left|j_{a}\right| \leq\left|\hat{j}_{a}\right|$, there will be exactly $n^{m}$ index vectors $\mathbf{j}$ for each maximum index vector $\hat{\mathbf{j}}$, that is, exactly the same number as components of an $m$ th order tensor defined on an $n$-dimensional space. In addition, the number of maximum index vectors will be, clearly, equal to the total number of combinations of covariant and contravariant indices for an $m$ th order tensor.

The above equality, thus, permits the following correspondence: To each contravariant index $j_{a}$ we assign a positive sign to the respective component of $\hat{\mathbf{j}}$; to each covariant index $j_{b}$ we assign a negative sign to the respective component of $\hat{\mathbf{j}}$; and, as the index vector $\mathbf{j}$ varies in $J_{(m)}$ subject to the conditions $\operatorname{sign} j_{a}=\operatorname{sign} \hat{j}_{a}$ and $0<\left|j_{a}\right| \leq\left|\hat{j}_{a}\right|$, we assign to each $\mathbf{j}$ one of the respective components of the mixed tensor corresponding to $\hat{\mathbf{j}}$. All the $2^{m} n^{m}$ index vectors in $J_{(m)}$ thus specify all the possible covariant, contravariant, and mixed components of a given $m$ th order tensor defined on an $n$-dimensional coordinate space.

In particular, if $t^{\mathrm{i}}$ are components of arbitrary variance (that is, some of the components of the index vector may be negative) of a given tensor $T$, then $t^{-\mathbf{i}}=t_{\mathbf{i}}$ and the form of the transformation law (1) remains valid for this generalization if we also define $x_{-\mathbf{i}}^{-\mathbf{r}}=x_{\mathbf{r}}^{\mathbf{i}}$. Thus, (1) may be written as either $t^{\mathbf{r}}=t^{\mathbf{i}} x_{\mathbf{i}}^{\mathbf{r}}$ or $t_{\mathbf{s}}=t_{\mathbf{j}} x_{\mathbf{s}}^{\mathbf{j}}$ regardless of the variance of the components.

Defining $g_{\mathbf{i j}}=g_{i_{1} j_{1}} g_{i_{2} j_{2}} \ldots g_{i_{m} j_{m}}, g^{\mathbf{i j}}=g^{i_{1} j_{1}} g^{i_{2} j_{2}} \ldots g^{i_{m} j_{m}}$ and $\delta_{\mathbf{j}}^{\mathbf{i}}=g^{\mathbf{i k}} g_{\mathbf{k j}}=\delta_{j_{1}}^{i_{1}} \delta_{j_{2}}^{i_{2}} \ldots \delta_{j_{m}}^{i_{m}}$, where the indices $i_{a}$ and $j_{a}$ may be either positive or negative, the corresponding "covariant" Christoffel Connection will be given by $\Gamma_{\mathbf{i} \mid \mathbf{j} k}=g_{\mathbf{i h}} \Gamma_{\cdot \mathbf{j} k}^{\mathbf{h}}$. Using the identity

$$
\frac{\partial g_{\mathbf{i k}}}{\partial x^{j}}=\left(\Gamma_{i_{1} k_{1}}+\Gamma_{k_{1} j i_{1}}\right) g_{i_{2} k_{2}} \ldots g_{i_{m} k_{m}}+\ldots+\left(\Gamma_{i_{m} j k_{m}}+\Gamma_{k_{m} j i_{m}}\right) g_{i_{1} k_{1}} \ldots g_{i_{m-1} k_{m-1}},
$$

the expression (2) for the covariant derivative of a tensor of arbitrary order may be written in either of the forms

$$
\left.t^{\mathbf{r}}\right|_{s}=\frac{\partial t^{\mathbf{r}}}{\partial x^{s}}+t^{\mathbf{p}} \Gamma_{\cdot \mathbf{p} s}^{\mathbf{r}} \quad \text { or }\left.\quad t_{\mathbf{i}}\right|_{s}=\frac{\partial t_{\mathbf{i}}}{\partial x^{s}}-t_{\mathbf{k}} \Gamma_{\cdot \mathbf{i} s}^{\mathbf{k}}
$$

regardless of variance. The similar generalization of the integrability conditions (3) for tensors of arbitrary mixed variance is obvious. 


\section{Order Transformations}

The identity $g_{\mathrm{ij}} t^{\mathbf{j}}=t^{-\mathbf{i}}$ which follows from the results of the last section suggests the definition $\left(t^{\mathbf{i}}\right)^{-1}=t^{-\mathbf{i}}$ or, generalizing the latter, the definition $t^{\mathbf{i}}=\left(t^{\mathbf{j}}\right)^{\omega}=t^{\omega \mathbf{j}}$, where $\boldsymbol{\omega}$ is a rotation operator in $J_{(m)}$ subject to the restriction $0<\left|i_{a}\right| \leq n$. Thus, a variance transformation of the components of a tensor $T$ on $G$ can be considered equivalent to a restricted orthogonal transformation of $\hat{\mathbf{j}}$ in the index space $J_{(m)}$.

By removing the restriction, a more general orthogonal transformation $\boldsymbol{\alpha}$ can be defined which can be called a decreasing order transformation, that is, $\boldsymbol{\alpha}$ will be a transformation which rotates $\hat{\mathbf{j}}$ into a subspace $J_{(\nu)}$ of $J_{(m)}$ and, correspondingly, transforms the tensor $T_{(m)}$ into $T_{(\nu)}$. If $\hat{\boldsymbol{\alpha}}$ is a maximum order transformation, that is, one which transforms $\hat{\mathbf{j}}$ into a zero vector at the origin of $J_{(m)}$, then clearly there will be the same total number of such possible maximum order transformations $\boldsymbol{\alpha}_{(i)}$ as there are invariants of the tensor $T$.

In particular, the scalar $t^{\mathbf{0}}=t^{\mathbf{i}} t^{-\mathbf{i}}=t^{\mathbf{i}} t_{\mathbf{i}}=t^{i, i_{2} \cdots i_{n}} t_{i_{1} i_{2}} \cdots i_{n}$ is the only invariant possessed by tensors of all orders (and thus might be called the "fundamental quadratic invariant"). Just as contraction of a tensor is indicated in the ordinary notation by summation over a pair of indices (one covariant, one contravariant), contraction in this new notation results in a tensor $t^{\mathbf{i}(\nu)}=\Sigma_{i_{k}, i_{h}} t^{\mathbf{i}(n)}$, subject to the restriction $i_{k}+i_{h}=0$, with $k, h$ fixed, where $\nu=n-2$. The reduction in dimension of the index space results from, first, restricting the index vector to the hypersurface defined by $i_{k}+i_{h}=0$ and, second, summing the components of $t^{\mathrm{i}}$ (and assigning each sum to the corresponding sum of the index vectors) over the values of the index $i_{k}$; thus, the dimension of the index space is reduced by two.

Further consideration of general order transformations should be of considerable value in the theory of groups as used in quantum mechanics, in the study of irreducible tensors, and in determining the invariants of tensors of arbitrary order. If such general order transformations were unrestricted they would clearly lead to tensors of more general type, that is, tensors represented by a set of components defined, not as functions of a point in a single space, but as functions of $M$ points in $M$ spaces having possibly different dimensions.

Boulder, Colo.

(Paper 64B2-27) 